\title{
3 Research Square

\section{An Investigation of Risk Factors Affecting Short Term Mortality of Patients Referring to the Hospital Emergency Department}

\section{Elham Peyravi}

Shiraz University of Medical Sciences

Hadid Hamrah

Shiraz University of Medical Sciences

Mohammad Sadegh Masoudi

Shiraz University of Medical Sciences

Milad Ahmadi Marzaleh

Shiraz University of Medical Sciences https://orcid.org/0000-0003-1743-0093

Mahmoudreza Peyravi ( $\nabla$ peyravi110@gmail.com )

Shiraz University of Medical Sciences

Research article

Keywords: Short term mortality, Emergency department, Hospital, Risk

Posted Date: November 17th, 2020

DOI: https://doi.org/10.21203/rs.3.rs-108213/v1

License: (c) (i) This work is licensed under a Creative Commons Attribution 4.0 International License.

Read Full License 


\section{Abstract}

Background and Objective: One of the causes of short-term mortality in patients is the lower quality of services provided by hospital emergency departments. Given the particular importance of the hospital emergency system and the presence of numerous problems, as well as short term mortality rates in hospitals, this study aimed to investigate the risk factors affecting short term mortality of patients presenting to the Emergency Department at Nemazi Hospital in Shiraz, Fars province in 2019.

Methods: This is a retrospective study with a case control-analytical design. The sample size was 768 subjects. In the present study, the emergency department overcrowding was measured by the NEDOCS (National Emergency Department Overcrowding Scale) criterion. The severity of the disease was also evaluated based on the level of the triage of patients through the Emergency Severity Index (ESI) system and vital signs.

Results: With each year increase in age, the chance of short-term mortality increases by $0.8 \%$. People with 02 sat $\%<90 \%$ are 7.3 times more likely to experience short term mortality in an emergency department compared to people with 02 sat $\%>90 \%$. A significant relationship was noted between short term mortality and SBP (systolic blood pressure) in the hospital's emergency department. It was also found out that as the triage score increases, short term mortality decreases significantly. As hospital stay increases, the chance of the patients' mortality decreases by $0.5 \%$.

Conclusion: The percentage of arterial blood oxygen saturation, systolic blood pressure, respiration rate per minute, triage score, the way the patient arrives at the hospital, working shifts, hospitalization duration, age, and comorbidities were regarded as the risk factors for short term mortality. Therefore, promoting professional knowledge and skills of nurses and physicians in the hospitals' emergency department and up-to-dating and reviewing emergency protocols as well as similar research can greatly help reduce short term mortality in the hospital's emergency department.

\section{Introduction}

Hospital emergency systems are seen as critical components of the health system. If these systems' operations are disrupted such that they cannot perform their duties properly, one should expect crises to appear with the patient's risk of loss of life. Identifying the effective factors and causes of short-term mortality and encountering with their etiological factors are the most appropriate strategies to reduce short term mortality before the patient is admitted at the hospital departments. As much as 45 to $60 \%$ of patients' mortality occurs during the first 24 hours after admission to the hospital's emergency department (1-3). Many studies have demonstrated that cardiovascular diseases, traffic incidents, trauma, and cancer are the most important causes of mortality in hospital emergency wards. Of course, the cause of mortality varies in various geographical areas (4). The impact of death risk factors can also vary over time. Some risk factors may be more important in the first several hours of admission to the hospital's emergency ward, while other risk factors may have a greater impact on mortality in the 
following hours and days. Because the impact of risk factors is likely to vary considering the time elapsed after the symptoms occur (or after being admitted to the hospital emergency department), this study aimed to investigate the related risk factors leading to death in four time intervals: 0-6 hours, 6-12 hours, 12-24 hours and 24-72 hours, in line with the time allotted for the hospitalization of patients at different levels of emergency (5-7).

Previous studies suggested that the medical emergency team could reduce the deaths of patients with unexpected cardiac arrest based on their abnormal clinical observations; however, the relationship between any abnormal clinical observations and a subsequent increase in death had not been investigated. Two cases of the most prevalent abnormal clinical occurrences before the patients' condition deteriorated were arterial oxygen saturation (51\%) and hypotension (17.3\%). As a result, there were six clinical observations that anticipated death. These observations included reduced level of consciousness (GCS) by 2, onset of coma, hypotension ( $90 \mathrm{mmHg}>$ ), respiratory rate less than 6 beats per minute, reduced arterial oxygen saturation $(90 \%>)$, and bradycardia (30 per minute) (8).

Short term mortality is one of the harbingers of low medical services quality in pre-hospital and hospital emergency departments $(9,10)$. Hospital emergency department, as the first line of treatment for patients, is of special importance. The mortality rate of each emergency department at any hospital is closely related with medical equipment, nursing staff and number of medical employees, as well as patients' demographic characteristics $(1,11)$. Scant studies have been done in Iran and the world to recognize the factors affecting short term mortality of patients in hospital emergency departments. Considering the special importance of the hospital emergency system and the presence of numerous problems, as well as the incidence of many short tern mortalities in Iranian hospitals, especially Fars province, we aimed to investigate the risk factors affecting short term mortality of patients presenting to the emergency department at the Nemazi Hospital in Shiraz, Iran, in the year 2019.

\section{Methods}

This study is a retrospective study using a case control-analytical design.

\section{Samples}

In this study, the subjects comprised of patients presenting to the emergency department at Nemazi Hospital in Shiraz. The sample size amounted to 768 subjects selected by random sampling. 384 patients who were dead and 384 alive patients who were admitted in ED less than 72 hours.

Inclusion criteria included a stay of less than 72 hours in the emergency department and a homogeneous selection of both sexes.

Exclusion criteria included people without underlying diseases, age under 18 years, and incomplete patient files.

\section{Data collection}


In this study, the death risk factors in the first hours of admission were assumed to be as follows: the first visit of the patient by the physician, crowding rate at the emergency department, patient's abnormal vital signs and triage level according to ESI criteria when admitted to the hospital's emergency department, though age, gender, comorbidities, duration of hospitalization, and the way the patient is transferred to the emergency room could be considered as the risk factors, both in the short term and in the days to come. The patient's physiological signs including heart rate (HR), body temperature, time of first visit, the way the patient arrives at the hospital, time and day of admission, systolic blood pressure (SBP), as well as respiratory rate per minute (RR) were also evaluated.

In the present study, the emergency department's overcrowding rate was measured by NEDOCS (National Emergency Department Overcrowding Scale) which was completed by the patient care nurse. The severity of the disease was measured based on the level of the triage of patients via the ESI system. The ESI triage system has 5 levels based on the severity of the disease and the need or lack of need to an emergency treatment, prioritized by the triage nurse within 2-5 minutes after the patient is admitted to the department. The guideline on the patient's comorbidity index (Charlson Comorbidity Index (CCI)) was completed as follows: if the patient has no underlying disease, a CCl weight of zero is assigned; if $\mathrm{s} / \mathrm{he}$ has a heart condition, suffering from environmental, stroke, asthma, diabetes, arthritis, weight 1 is considered; in the case of kidney disease or cancer, weight 2, and liver cirrhosis, weight 3 are regarded. All analyses were performed separately in four time intervals of $0-6,6-12,12-24,24-72$ hours.

\section{Data analysis}

Data were analyzed in data collection forms which included physiological characteristics, triage, NEDOCS, and $\mathrm{CCl}$. Because the response variable involves two states of life and death, logistic regression was used to analyze the data. In cases where the frequency was low at some levels, Fisher's Exact Test was used instead of logistic regression. In cases where the Fisher's Test was used, it was only possible to report the significance rate ( $P$ value), and in cases where logistic regression was applied, the odds ratio was also obtained. SPSS software version 23 was used for statistical analysis.

\section{Ethical considerations}

Patients' data not involving names and surnames were taken from the HIS system of Shiraz Nemazi Hospital's emergency department based on the general outlines of the ICD Book as the approval for the study was taken from the Ethics Committee. Because of the confidentiality and vitality of the information, an informed consent form was obtained from the subjects and then completed while other ethical considerations including anonymity of the forms, protection of personal information and provision of data on the study to patients and nurses were observed.

\section{Results}

The sample size was 768 patients with a mean age of 66 years. $50 \%$ of the subjects were male. The effect of age on the patients' short-term mortality was close to a significance level $(\mathrm{Sig}=0.06)$. with the 
increase of one year to the subject's age, the chance of death increased by $0.8 \%$, and as the triage score rose, short term mortality decreased significantly. However, not all the survivors had a triage level of 1 , while $49.6 \%$ of the deceased had a triage level of 1 . In the meantime, $0.5 \%$ of the living people had triage level 4 and not all the deceased had a triage level of 4 . It should be borne in mind that not all of the living and the deceased patients had a triage level of 5 , as this issue confirms the principles of hospitalization of patients to be observed in the emergency department at Nemazi Hospital $(\mathrm{Sig}=0.00)$.

A statistically significant relationship was found between short term mortality and an increase of one unit in $\mathrm{CCl}$ (Charlson Comorbidity Index) (Sig = 0.00), so that by increasing one unit of the patient's CCl score, the chance of mortality increased by 3.8 times (Odd's ratio $=3.8$ ).

NEDOCS was 6 on all days, indicating the highest overcrowding level, so it cannot be considered a variable because it is homogeneous or measures its relationship with short term mortality either.

The variable of the patient's first visit by the physician was found to be inversely effective on the patients' short-term mortality ( $\mathrm{Sig}=0.001)$. The chance of death declined by $20 \%$, as one of the four defined scheduling levels increased. This result is probably due to appropriate prioritization of the patients' level of severity by Nemazi hospital's emergency physicians; this means that the time for visiting the patients is determined based on their triage levels and the disease severity, so patients with less disease severity take subsequent priority and are visited later by the relevant physician. A significant relationship was also noted between the duration of stay at the hospital and patients' short-term mortality (Sig $=0.001)$. Also, as the duration of hospitalization increased, the chance of patients' death decreased by $0.5 \%$.

A significant relationship was observed between short term mortality and SBP (systolic blood pressure) in the hospital emergency department (Sig $=0.001)$. Those survived all had SBPs $>90$, whereas $18 \%$ of people undergoing short term mortality had SBP $<90 \%$. There was a statistically significant relationship between RR (respiratory rate per minute) and patients' short-term mortality in the hospital emergency department $(\mathrm{Sig}=0.015)$. All living subjects had RR in a normal range of 5-36, while in people with short term mortality, 2 people had RR $<5$ and 5 had RR $>36$. The variable of body temperature in the normal range was found to have a significant effect on short term mortality $(\mathrm{Sig}=0.001) .5 .7 \%$ of the living subjects had Temp $<35$, whereas in general $1.8 \%$ of the people with short term mortality had normal temperature. This indicates that Temp will not predict short term mortality in the abnormal range. There was also a significant relationship between 02 Sat\% (percentage of arterial blood oxygen saturation) and short-term mortality in the emergency department, as people with 02 sat $\%<90 \%$ had a 7.3 chance of experiencing death in the emergency department, compared to people with $02 \mathrm{Sat} \%>90 \%(\mathrm{Sig}<0.001)$. It was found out that that the way patients were transferred at the hospital was related with short term mortality (Sig $=0.001) .55 .2 \%$ of the deceased and $18.5 \%$ of the living patients had been transferred to the hospital by ambulance. $71.3 \%$ of the survivors and $24.6 \%$ of the patients with short term mortality had presented on their own accord to the emergency department of Nemazi Hospital. Therefore, there was a statistically significant relationship between short term mortality and the way the patient was taken to the hospital. 
There was a significant relationship between the patients' arrival time at the hospital's emergency department in terms of working shifts and short-term mortality $(P$ value $=0.06)$. with an increase of one class to working shift, the chance of death increased by $18 \%$.

The results of the relationship between different criteria and short-term mortality at different time intervals are provided in Table 1.

Table 1. The relationship between different criteria and short-term mortality at different time intervals

\begin{tabular}{|c|c|c|c|c|c|}
\hline \multirow[t]{2}{*}{ eria } & \multirow[t]{2}{*}{$\begin{array}{l}\text { P- } \\
\text { Value }\end{array}$} & \multicolumn{4}{|c|}{$\begin{array}{l}\text { Significance (P-value) at time } \\
\text { intervals (hours) }\end{array}$} \\
\hline & & $0-6$ & 6-12 & $12-24$ & $24-72$ \\
\hline ge level and short-term mortality & $<001 / 0$ & $<001 / 0$ & $<001 / 0$ & $<001 / 0$ & $<001 / 0$ \\
\hline and short-term mortality & $<05 / 0$ & $<05 / 0$ & $<05 / 0$ & $<05 / 0$ & $<05 / 0$ \\
\hline $\begin{array}{l}\text { rlson Comorbidity Index and short- } \\
\text { I mortality }\end{array}$ & $<001 / 0$ & $<001 / 0$ & $<001 / 0$ & $<001 / 0$ & $<001 / 0$ \\
\hline $\begin{array}{l}\text { time visit by the physician and short- } \\
\text { I mortality }\end{array}$ & $<001 / 0$ & $>001 / 0$ & $>001 / 0$ & $<001 / 0$ & $<001 / 0$ \\
\hline $\begin{array}{l}\text { th of stay at the hospital and short- } \\
\text { I mortality }\end{array}$ & $<001 / 0$ & $>05 / 0$ & $>05 / 0$ & $>05 / 0$ & $>05 / 0$ \\
\hline $\begin{array}{l}\text { when patients arrive at the hospital } \\
\text { erms of week days and short-term } \\
\text { tality }\end{array}$ & $>05 / 0$ & $>05 / 0$ & $>05 / 0$ & $>05 / 0$ & $>05 / 0$ \\
\hline $\begin{array}{l}\text { rt rate and short-term mortality } \\
\text { rate }\end{array}$ & $>05 / 0$ & $>05 / 0$ & $>05 / 0$ & $>05 / 0$ & $>05 / 0$ \\
\hline $\begin{array}{l}\text { Jlic blood pressure and short-term } \\
\text { tality }\end{array}$ & $<001 / 0$ & $>05 / 0$ & $<001 / 0$ & $<001 / 0$ & $<001 / 0$ \\
\hline jiratory rate and short-term mortality & $<001 / 0$ & $>05 / 0$ & $>05 / 0$ & $>05 / 0$ & $<001 / 0$ \\
\hline $\begin{array}{l}\text { temperature and short-term } \\
\text { tality }\end{array}$ & $<001 / 0$ & $>05 / 0$ & $>05 / 0$ & $<001 / 0$ & $>05 / 0$ \\
\hline $\begin{array}{l}\text { :entage of arterial blood oxygen } \\
\text { ration and short-term mortality }\end{array}$ & $<001 / 0$ & $>05 / 0$ & $<001 / 0$ & $<001 / 0$ & $<001 / 0$ \\
\hline $\begin{array}{l}\text { way the patient has been taken to the } \\
\text { sital and short-term mortality }\end{array}$ & $<001 / 0$ & $<001 / 0$ & $<001 / 0$ & $<001 / 0$ & $<001 / 0$ \\
\hline $\begin{array}{l}\text { When patients arrive at the hospital } \\
n \text { patients arrive at the hospital in } \\
\text { is of }\end{array}$ & $<001 / 0$ & $>05 / 0$ & $>05 / 0$ & $>05 / 0$ & $<001 / 0$ \\
\hline
\end{tabular}

\section{Discussion}


The older the people are, the more likely they die of short-term mortality. Therefore, more care should be taken of the elderly. Compared to younger individuals, the elderly patients are at more risk of adverse outcomes, and they should be paid more attention in the emergency department when a risk assessment is performed. A study by Schultz et al. (2019) demonstrated that 139 middle-aged patients and 596 elderly ones died in 7 days. A 7-day mortality rate in the elderly was proved to be higher(12). A study by Nørgaard Bech et al. suggested that old age was an important risk factor for short-term mortality(5). The research by Gentile et al. to identify the risk factors for short-term mortality among the elderly three months after presenting to the emergency department revealed that there were two independent predictors of mortality: malnutrition and the score scale of the cumulative disease for the elderly. Malnutrition was found to be the strongest independent risk factor for short-term mortality(13). This study was conducted in line with the present research. More special care for the elderly seems necessary because of their increased chance of mortality.

Studies have also demonstrated that abnormal vital parameters and the severity of the disease on admission in the first hours and days are associated with mortality $(5,11)$. A study by Bruun Kristensen et al. (2015) indicated that a seven-day mortality rate varied for $1.8 \%$ of emergency department patients, $2.2 \%$ of ambulance patients, and $5.7 \%$ of emergency care unit patients. The best blood pressure was 95 to $119 \mathrm{~mm} \mathrm{Hg}$ in the emergency department, 103 to $120 \mathrm{~mm} \mathrm{Hg}$ in the ambulance and 101 to $115 \mathrm{~mm} \mathrm{Hg}$ in the emergency care unit. Systolic blood pressure does not solely suffice to identify high-risk patients. However, if the threshold is determined, the systolic blood pressure threshold of 100 to $110 \mathrm{~mm} \mathrm{Hg}$ is probably more suitable than $90 \mathrm{mg} \mathrm{Hg}(3)$. Also, the higher or lower the respiratory rate, the higher the risk of short-term mortality.

In the present study, the variable of body temperature in the normal range was found to have a significant impact on short-term mortality. However, a study by Nørgaard Bech et al. indicated that body temperature was not found to be associated with short-term mortality when the patient was admitted to the emergency unit(5). This might have been due to the specific characteristics of patients that requires more investigation.

A significant relationship was found between the duration of the patients' stay at the hospital and their short-term mortality. Furthermore, as the duration of the patients' stay at the hospital increased, the chance of patients' death decreased by $0.5 \%$. Because the hospital provides better care for the patient, it is suggested that patients stay at the hospital until they recuperate completely.

The time the patients arrive in the hospital's emergency department in terms of days of the week did not have any statistically significant relationship with short-term mortality. There was a close relationship between the patients' arrival time in the hospital emergency department in terms of working hours and premature death. Increasing a class to a working shift increases the probability of death by $18 \%$. A rise in the probability of morality may be due to the fact that in the evening and night shifts, the official in charge of the emergency unit is not present. 
However, the study by Nørgaard Bech et al. suggested that the severity of the disease by the time the patient is admitted, transfer by the emergency physician and abnormal vital parameters were associated with a 0-2 rate of mortality(5).

A study entitled "Causes of death in the emergency department; a short report" was conducted by Alaei et al. in Iran in 2014. The most prevalent causes of death were respiratory disease, heart disease and trauma. Respiratory, cardiovascular and trauma diseases are the most common causes of death in the emergency department, so proper planning, training of staff and provision of advanced equipment for emergency departments can be very effective in reducing the number of deaths(14). Comorbidity is also one of the causes of short-term mortality. Therefore, an exact background must be taken from the person at the time $\mathrm{s} / \mathrm{he}$ is admitted.

In the present study, there was no statistically significant relationship between short-term mortality and the time the patient was admitted to the hospital in terms of working shifts in any of the time intervals. It is rational to suggest that the personnel's working shifts are not associated with death rates. The results by Guttman et al. suggested that the risk of complications had increased with the mean duration of stay, and the proportion of short-term mortality had increased by the same ratio. Patients who had been discharged from the emergency department earlier were not exposed to short-term complications(15).

Short-term mortality was significantly related with an increase in one unit based on CCl (Charlson Comorbidity Index). Thus, as one unit is added to the patient's $\mathrm{CCl}$ score, the chance of death increases by 3.8 times. Comorbidity is also one of the causes of short-term mortality. Therefore, an exact background must be taken from the person at the time s/he is admitted. Results of a study by Esteban et al. proved that the lowest mortality was associated with basal dyspnea. The highest mortality rate was associated with basal dyspnea, use of assistive devices, dysfunctional respiration by the time of arrival at the emergency room, with Glasgow score being less than 15 (16). Many studies suggested that the way the patient was transferred to the hospital(17) and the first-time visit of patients by the physician in the hospital emergency department were associated with the outcome of the patients' fate in the pre-hospital emergency department(18-20).

Short-term mortality occurs in many Iranian hospitals and it is because of various factors including the patient him/herself, the healthcare team, and the health system. Improving the professional knowledge and nurses' and physicians' skills in the emergency departments at hospitals and pre-hospitals, as well as strengthening and reviewing emergency guidelines, can help reduce the mortality rates. Of course, there may be other hidden issues; however, health-policy makers and directors should consider programs to help improve the hospital and pre-hospital services which aim at reducing the patients' deaths.

That the way the patients arrives at the hospital is significantly related to the patients' short-term mortality $(\mathrm{Sig}=0.00)$ is to their benefit as it may help prevent such death rates. This suggests that patients who had been taken to the hospital were in a better condition than those who had presented to Nemazi Hospital on their own accord(17). 
This study investigated the risk factors associated with short-term mortality in Shiraz Nemazi Hospital. Various factors were found to have affected the short-term mortality. Hospital and emergency department managers must consider various factors, including patient-related factors, emergency department, and the pre-hospital and hospital emergency system in order to reduce the short-term mortality. The following suggestions are provided in line with the present study:

1. It is suggested that special training courses should be held to prepare emergency department personnel to improve their performance so that short-term mortality is reduced.

2. It is suggested that guidelines and instructions should be provided for sensitive and vulnerable groups.

3. It is advised that needs assessment should be done to equip the hospitals' emergency department and pre-hospitals services for rapid response to patients.

4. It is recommended that a committee should be established to investigate short-term mortality at hospitals and medical universities of the country.

That the study only incorporated one hospital was a limitation. The findings at other hospitals in Fars province or different provinces of Iran may be different from the finding of Nemazi Hospital.

\section{Conclusion}

Arterial blood oxygen saturation, systolic blood pressure, respiration rate per minute, triage score, patient's arrival time, working shift, hospitalization time, age, and comorbidities were considered to be the risk factors for short-term mortality. Therefore, improving the nurses and physicians' professional knowledge and skills in the emergency department and strengthening and reviewing emergency guidelines as well as similar research can help reduce short-term mortality in the hospital emergency department. Further studies in this field will complement the findings of the future research. We, in the end, appreciate, the president, managers and staff at Nemazi Hospital in Shiraz, who helped us with conducting this research.

\section{Declarations}

Acknowledgement: The management, managers and staff of Nemazi Hospital in Shiraz, who paved the way for this study, are thanked and appreciated.

\section{Footnotes}

Authors' Contribution: Elham Peyravi, Hadid Hamrah, Mohammad Sadegh Masoudi, Mahmoudreza Peyravi, and Milad Ahmadi Marzaleh designed this study together and did the literature review. Mahmoudreza Peyravi was responsible for diagnosis and Elham Peyravi, Hadid Hamrah, Mohammad Sadegh Masoudi, Mahmoudreza Peyravi, and Milad Ahmadi Marzaleh were responsible for collecting information. Elham Peyravi, Hadid Hamrah, Mohammad Sadegh Masoudi, Mahmoudreza Peyravi, and Milad Ahmadi Marzaleh analyzed data and wrote the report together. 
Conflict of Interest: Nil.

Informed Consent: Informed consent was obtained from all individual participants included in the study.

Funding/Support: This project was supported in part by a grant from Shiraz University of Medical Sciences with the code 14473.

Financial Disclosure: Nil.

Ethical Approval: The researchers presented approval obtained from Shiraz University of Medical Sciences with code 14473 to the research units.

\section{References}

1. Mahmoudi GA, Astaraki P, Anbari K. Epidemiolgical survey of mortality rate in patients admitted to Shohada Hospital of Khorramabad in 2011. Iranian Journal of Forensic Medicine. 2014;20(1):393400.

2. Hoikka M, Silfvast T, Ala-Kokko TI. Does the prehospital National Early Warning Score predict the short-term mortality of unselected emergency patients? Scandinavian journal of trauma, resuscitation and emergency medicine. 2018;26(1):48.

3. Kristensen AKB, Holler JG, Mikkelsen S, Hallas J, Lassen A. Systolic blood pressure and short-term mortality in the emergency department and prehospital setting: a hospital-based cohort study. Critical Care. 2015;19(1):158.

4. Wall MM, Huang J, Oswald J, McCullen D. Factors associated with reporting multiple causes of death. BMC Medical Research Methodology. 2005;5(1):4.

5. Bech CN, Brabrand M, Mikkelsen S, Lassen A. Risk factors associated with short term mortality changes over time, after arrival to the emergency department. Scandinavian journal of trauma, resuscitation and emergency medicine. 2018;26(1):29.

6. Pirneskoski J, Kuisma M, Olkkola KT, Nurmi J. Prehospital national early warning score predicts early mortality. Acta Anaesthesiologica Scandinavica. 2019;63(5):676-83.

7. Darioli V, Taffé P, Carron P-N, Dami F, Vallotton L, Yersin B, et al. Evaluation of the discriminative performance of the prehospital National Advisory Committee for Aeronautics score regarding 48-h mortality. European Journal of Emergency Medicine. 2019;26(5):366-72.

8. Murray CJ, Lopez AD. Global mortality, disability, and the contribution of risk factors: Global Burden of Disease Study. The lancet. 1997;349(9063):1436-42.

9. Dubois RW, Rogers WH, Moxley JH, Draper D, Brook RH. Hospital Inpatient Mortality: Is It a Predictor of Quality? 1991.

10. Takahashi M, Kohsaka S, Miyata H, Yoshikawa T, Takagi A, Harada K, et al. Association between prehospital time interval and short-term outcome in acute heart failure patients. Journal of cardiac failure. 2011;17(9):742-7. 
11. Marino R, Magrini L, Orsini F, Russo V, Cardelli P, Salerno G, et al. Comparison between soluble ST2 and high-sensitivity troponin I in predicting short-term mortality for patients presenting to the emergency department with chest pain. Annals of laboratory medicine. 2017;37(2):137-46.

12. Schultz M, Rasmussen LJH, Carlson N, Hasselbalch RB, Jensen BN, Usinger L, et al. Risk assessment models for potential use in the emergency department have lower predictive ability in older patients compared to the middle-aged for short-term mortality-a retrospective cohort study. BMC geriatrics. 2019;19(1):134.

13. Gentile S, Lacroix O, Durand A, Cretel E, Alazia M, Sambuc R, et al. Malnutrition: a highly predictive risk factor of short-term mortality in elderly presenting to the emergency department. The journal of nutrition, health \& aging. 2013;17(4):290-4.

14. Faridaalaee G, Nikzad F, Rahmani SH. Cause of Death in Emergency Department; a Brief Report. Iranian Journal of Emergency Medicine. 2015;2(1):45-8.

15. Guttmann A, Schull MJ, Vermeulen MJ, Stukel TA. Association between waiting times and short term mortality and hospital admission after departure from emergency department: population based cohort study from Ontario, Canada. Bmj. 2011;342:d2983.

16. Esteban C, Arostegui I, Garcia-Gutierrez S, Gonzalez N, Lafuente I, Bare M, et al. A decision tree to assess short-term mortality after an emergency department visit for an exacerbation of COPD: a cohort study. Respiratory research. 2015;16(1):151.

17. Peyravi M, Örtenwall P, Khorram-Manesh A. Can Medical Decision-making at the Scene by EMS Staff Reduce the Number of Unnecessary Ambulance Transportations, but Still Be Safe? PLoS currents. $2015 ; 7$.

18. Peyravi M, Khodakarim S, Örtenwall P, Khorram-Manesh A. Does temporary location of ambulances ("fluid deployment") affect response times and patient outcome? International journal of emergency medicine. 2015;8(1):37.

19. Boulanger J, Lindsay M, Gubitz G, Smith E, Stotts G, Foley N, et al. Canadian stroke best practice recommendations for acute stroke management: prehospital, emergency department, and acute inpatient stroke care, update 2018. International Journal of Stroke. 2018;13(9):949-84.

20. Ko J, Zhu S, Alabaster A, Wang J, Sax DR. Prehospital Treatment and Emergency Department Outcomes in Young Children with Food Allergy. The Journal of Allergy and Clinical Immunology: In Practice. 2020. 